\title{
Analysis of TCGA data of differentially expressed EMT-related genes and miRNAs across various malignancies to identify potential biomarkers
}

\author{
KONSTANTINOS A. KYRITSIS ${ }^{1}$, MELPOMENI G. AKRIVOU ${ }^{1}$, \\ LEFKI-PAVLINA N. GIASSAFAKI ${ }^{1}$, NIKOLAOS G. GRIGORIADIS ${ }^{1,2}$ and IOANNIS S. VIZIRIANAKIS ${ }^{1,3,4}$ \\ ${ }^{1}$ Laboratory of Pharmacology, School of Pharmacy, Aristotle University of Thessaloniki, 54124 Thessaloniki; \\ ${ }^{2}$ Biogenea Pharmaceuticals Ltd., 54627 Thessaloniki; ${ }^{3}$ Functional Proteomics and Systems Biology Research Group at \\ AUTH (FunPATH) Research Group, KEDEK-Aristotle University of Thessaloniki, Balkan Center, 57001 Thessaloniki, \\ Greece; ${ }^{4}$ Department of Life and Health Sciences, University of Nicosia, CY-1700 Nicosia, Cyprus
}

Received September 8, 2020; Accepted November 30, 2020

DOI: $10.3892 /$ wasj.2020.77

\begin{abstract}
Tumor heterogeneity presents a hindering factor that leads to therapeutic failures and limits the improvement of clinical outcomes within the concept of precision medicine. This heterogenous characteristic provides the epithelial mesenchymal plasticity that is considered an advantage for cancer cell metabolism and genome function to be adjusted within the microenvironment, and also plays a role in the development of drug resistance and metastasis. To this respect, identifying druggable molecular targets that modulate signaling networks, which contribute to cancer cell heterogeneity, could provide innovative therapeutics with improved safety and efficacy profiles. The present study attempted to identify potentially druggable molecular targets that have been connected to the process of epithelial-to-mesenchymal transition (EMT). Towards this goal, gene and miRNA differential expression analyses were performed for cancer patients with 4 and 3 different tumor types, respectively, using data that were retrieved from The Cancer Genome Atlas (TCGA) program. Furthermore, the dbEMT 1.0 database was used to limit the results to differentially expressed molecular targets that have already been associated with EMT. The analysis resulted in the identification of multiple EMT-associated genes and miRNAs for all types of cancer, which, through pairwise comparisons, were separated into groups of common potential targets for different malignancies. Differential gene expression profiling by RT-qPCR analysis was also carried out for a number of
\end{abstract}

Correspondence to: Professor Ioannis S. Vizirianakis, Laboratory of Pharmacology, School of Pharmacy, Aristotle University of Thessaloniki, 54124 Thessaloniki, Greece

E-mail: ivizir@pharm.auth.gr

Key words: epithelial-to-mesenchymal transition, the cancer genome atlas, RNA-Seq, miRNA-Seq, meta-analysis, druggable targets, anticancer therapeutics selected genes and miR-21 in human cancer cell lines. Notably, EMT-associated homeobox B9 (HOXB9) and miR-137 were found to have a deregulated expression in all malignancies examined, thus increasing their potential as druggable targets for cancer therapy. Overall, the present study presents an approach that, through systematic in silico analysis, could lead to the selection of potential druggable biomarkers of broader utility for several tumor types, irrespective of their tissue of origin.

\section{Introduction}

Cellular and genomic heterogeneity in tumors involves the microenvironment, cancer stem cells and epithelial-to-mesenchymal transition (EMT) $(1,2)$. EMT constitutes a complex and dynamic biological process during which epithelial cells transdifferentiate towards a mesenchymal phenotype. Unlike epithelial cells, which are characterized by polarity and maintain firmly cell-to-cell adhesion contacts through cellular adhesion molecules (CAMs), mesenchymal cells display an increased mobility and loose organization within the extracellular matrix. EMT plays an important role in physiological processes, including organ formation during embryogenesis and tissue regeneration; however, its involvement has also been confirmed in tumor initiation, progression and metastasis. The transition can be triggered through various stimuli, including different factors of the tumor microenvironment (cytokines, growth factors, etc.), as well as immune responses, hypoxia and antitumor drug treatment. Notably, EMT is reversible, exhibiting plasticity, with mesenchymal cells being capable of converting back to an epithelial phenotype through a process known as mesenchymal-to-epithelial transition (MET). The combination of EMT and MET can lead to a mixed, dynamic population of cancer cells exhibiting both epithelial and mesenchymal characteristics that also promote circulating tumor cell (CTC) formation. This can result in the disruption of cellular adhesion and increased migratory and invasive capabilities, which can lead to metastasis $(3,4)$. 
The EMT phenotypic plasticity of tumor cells contributes to molecular and cellular heterogeneity, that leads to acquired drug resistance to cytotoxic or molecularly targeted therapy in clinical practice. Moreover, the differential pharmacological response limits the productivity and clinical outcomes of innovative therapeutic approaches (5-7). Moreover, the interplay of transcription (including the Snail, Twist and Zeb families) and epigenetic factors (e.g., the miR-200 family, miR-205, miR-203, miR-34 and miR-29b) drive the regulatory network program of EMT plasticity in cancer (6). It would be of interest if common molecular drivers in these EMT and MET processes that are deregulated in various types of tumors could be characterized; following clinical validation, biomarkers could be developed which may be used in cancer therapy.

Previously the authors characterized the expression levels of several epithelial markers, namely desmoglein 3 (DSG3), E-cadherin and $\beta$ - $/ \gamma$-catenins $(\beta-/ \gamma$-catenins) in monolayer (ML) and multicellular aggregates (MCAs) of the HSC-3 cell line (oral squamous carcinoma) in vitro, as well as in clinical samples of oral leukoplakia (OL) and oral squamous cell carcinoma (OSCC) in vivo (8). Of note, the downregulation of DSG3, E-cadherin and $\beta$ - $/ \gamma$-catenins was observed to be significantly associated with the grade of OL-dysplasia and OSCC samples (8). Furthermore, the switch of expression and potent perinuclear aggregation of DSG3 and $\gamma$-catenin were observed in both HSC-3 cells and OL/OSCC samples. These observations support the involvement of DSG3 and $\gamma$-catenin in the progression of oral epithelial cell malignancy. It was also suggested that these genes may serve as potential predictive biomarkers, along with E-cadherin and $\beta$-catenin, of the malignant transformation risk of oral dysplasia and the biological behavior (aggressiveness) of oral cancer, respectively (8).

In the present study, an in silico analysis was performed using RNA-Seq data from The Cancer Genome Atlas (TCGA) database (9), in order to identify genes that are involved in the EMT and MET processes, and that may serve as possible biomarkers and/or therapeutic targets in clinical practice. For this purpose, differential gene and microRNA (miRNA/miR) expression analyses were carried out between solid tissue normal (STN) and primary solid tumor (PST) samples from 4 different types of cancer (head and neck, prostate and breast cancer, and glioblastoma). The accurate separation of STN and PST samples into distinct clusters was confirmed using principal component analysis (PCA) based solely on the identified differentially expressed (DE) genes/miRNAs (Fig. 1). Additionally, dbEMT 1.0, a database containing EMT-related genes collected through extensive literature search (10), was used to further filter DE genes and miRNAs, and keep those that have been found to be involved in EMT and/or MET. On the whole, the present study identified and reported several DE and EMT/MET-related genes and miRNAs in various types of malignancies, whose potential in clinical utilization will be further evaluated by characterizing their expression levels in cell line EMT models and in clinical samples in the future.

\section{Materials and methods}

Cancer data. RNA-Seq and miRNA-Seq data from TCGA (9) were retrieved using TCGAbiolinks (11). Specifically, from the available pre-processed data types, gene and miRNA count data, derived from HTSeq software (12), were selected for 4 different types of cancer: i) Head-and-neck squamous cell carcinoma (TCGA-HNSC); ii) breast cancer (TCGA-BRCA); iii) prostate adenocarcinoma (TCGA-PRAD); and iv) glioblastoma multiforme (TCGA-GBM).

Differential expression analysis. To ensure the power of statistical testing, only STN and PST samples were selected to perform differential expression analysis for genes and miRNAs using DESeq2 (13).

Due to the lack of sufficient miRNA-Seq samples in TCGA-GBM, miRNA differential expression analysis was performed only for TCGA-BRCA, TCGA-HNSC and TCGA-PRAD.

A minimum threshold of 100 and 10 total number of counts was set to filter out genes and miRNAs with very low counts, respectively. Genes and miRNAs with an absolute log2 fold change (LFC) $>1$ and a false discovery rate (FDR) (14) adjusted P-value $<0.001$ were reported as statistically significant, DE targets. Variance-stabilizing transformation (VST) was applied to DE gene or miRNA expression values of all samples, followed by PCA. Both VST and PCA were performed using DESeq2 (13).

EMT-associated genes and miRNAs. Genes and miRNAs that have been associated with EMT were retrieved from dbEMT 1.0, a database containing EMT-related genes and miRNAs that were collected through extensive literature search (10). Comparisons of EMT targets with DE genes and miRNAs was performed with $\mathrm{R}$ statistical programming language. Venn diagrams were created using limma (15).

Survival analysis of DE genes and miRNAs. Survival analysis was performed on DE genes and miRNAs using clinical metadata from TCGA. Specifically, for each DE gene or miRNA, PST samples were assigned into 2 separate groups, depending on whether the target expression of each sample was higher (high expression) or lower (low expression) than the median. Kaplan-Meier analysis on the 2 groups was performed for each DE gene or miRNA and selected, statistically significant targets are reported in Kaplan-Meier survival curves. For the statistical analysis of DE genes (P-value <0.001) and miRNAs (P-value <0.1) the non-parametric log-rank test was used. An exception to this was miR-16-1 in HNSC cancer samples, with a P-value of $\sim 0.12$. Survival analysis was performed using a survival analysis package $(16,17)$ and survminer $(18)$.

Cell cultures, RNA isolation and RT-qPCR analysis. The established cell lines of human breast epithelial carcinoma MCF-7 (Cellosaurus, CVCL-0031) and MDA-MB-231 (Cellosaurus, CVCL-0062), as well as the human tongue squamous carcinoma HSC-3 (Cellosaurus, CVCL-1288) and keratinocyte HaCaT (Cellosaurus, CVCL-0038) cancer cells that are routinely used in the authors' laboratory were cultured as previously described $(19,20)$. Moreover, the RNA isolation, RT-cDNA synthesis, as well as the RT-qPCR analysis were carried out as previously described $(19,20)$. In brief, total RNA was extracted from cells using TRItidY G (Panreac, Applichem), quantified using a Nanodrop ND-100 
Table I. Number of genes and miRNAs identified as differentially expressed and EMT-associated in different malignancies.

\begin{tabular}{|c|c|c|c|c|c|}
\hline TCGA project ID & Type of malignancy & $\begin{array}{c}\text { Number of } \\
\text { DE genes } \\
(\text { LFC }>1 \text { and } \\
\text { FDR adjusted } \\
\text { P-value }<0.001)\end{array}$ & $\begin{array}{l}\text { Number of } \\
\text { DE Genes } \\
\text { reported by } \\
\text { dbEMT } 1.0\end{array}$ & $\begin{array}{c}\text { Number of } \\
\text { DE miRNAs } \\
(\text { LFC }>1 \text { and } \\
\text { FDR adjusted } \\
\text { P-value }<0.001)\end{array}$ & $\begin{array}{l}\text { Number of } \\
\text { DE miRNAs } \\
\text { reported by } \\
\text { dbEMT } 1.0\end{array}$ \\
\hline TCGA-HNSC & $\begin{array}{l}\text { Head and neck squamous } \\
\text { carcinoma }\end{array}$ & 2,509 & 37 & 255 & 6 \\
\hline TCGA-BRCA & Breast cancer & 4,227 & 73 & 271 & 10 \\
\hline TCGA-PRAD & Prostate cancer & 1,049 & 11 & 110 & 4 \\
\hline TCGA-GBM & Glioblastoma multiforme & 2,350 & 44 & & \\
\hline
\end{tabular}

DE, differentially expressed; EMT, epithelial-to-mesenchymal transition.
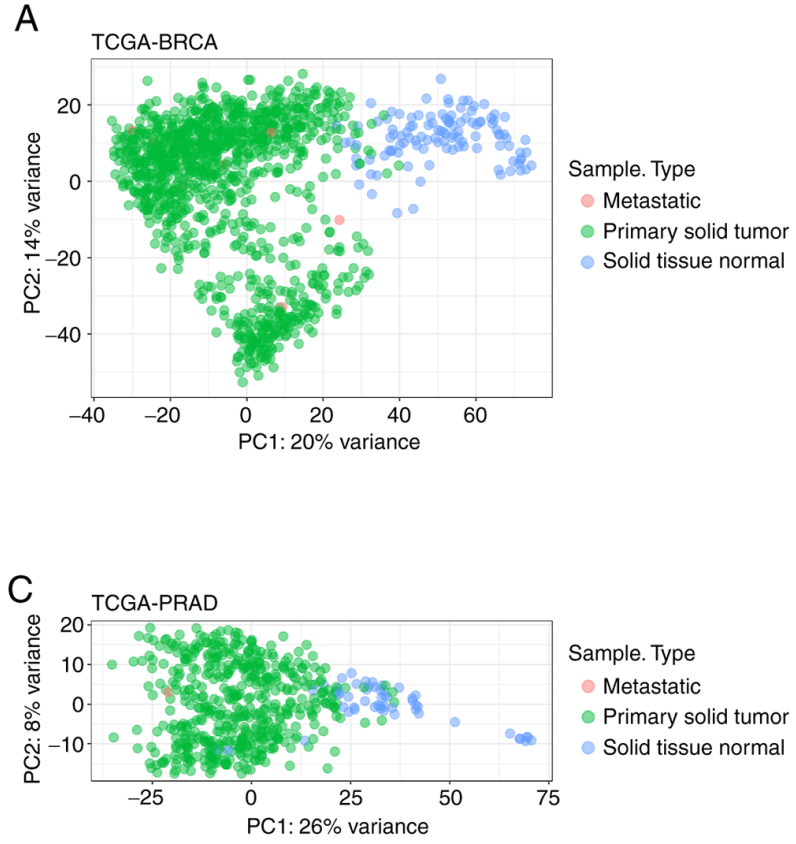
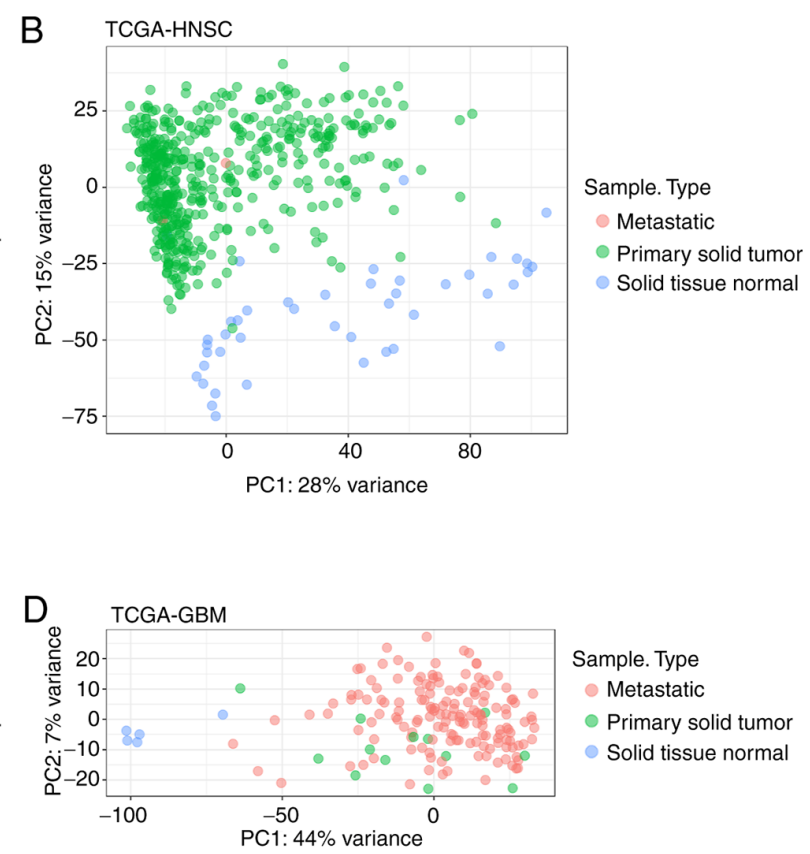

Figure 1. PCA of all samples from (A) breast cancer, (B) head and neck cancer, (C) prostate cancer and (D) glioblastoma using the expression values of differentially expressed genes. PCA was performed and scatterplots were created using DESeq2 (13). PCA, principal components analysis; TCGA, The Cancer Genome Atlas; BRCA, breast cancer; HNSC, head and neck squamous cell carcinoma; PRAD, prostate adenocarcinoma; GBM, glioblastoma multiforme.

Spectrometer and reverse transcribed into cDNA by applying the QuantiTect Reverse Transcription kit (Qiagen, Inc.). qPCR was performed on a 7500 Real-Time PCR System (Applied Biosystems; Thermo Fisher Scientific, Inc.) using KAPA SYBR $^{\circledR}$ FAST qPCR Master Mix (KAPA Biosystems) under optimized conditions: $95^{\circ} \mathrm{C}$ for 3 min followed by 40 cycles at $95^{\circ} \mathrm{C}$ for $3 \mathrm{sec}$ and $60^{\circ} \mathrm{C}$ for $20 \mathrm{sec}$. Primers designed and used during the present study were as follows: (PPARG forward, 5'-TCG-AGG-ACA-CCG-GAG-AGG-3' and reverse, 5'-CACGGA-GCT-GAT-CCC-AAA-GT-3'; HMGA2 forward, 5'-GAA-AAA-CGG-CCA-AGA-GGC-AG-3' and reverse, 5'-AGA-GCT-ATC-CTG-GAC-TCC-TCC-3';FOXM1 forward, 5'-ACC-GCT-ACT-TGA-CAT-TGG-AC-3' and reverse, 5'-GGG-AGT-TCG-GTT-TTG-ATG-GTC-3'; CAV-1 forward, 5'-CCC-AGG-GAA-ACC-TCC-TCA-CAG-3' and reverse, 5'-GGC-AGA-TAG-CAG-AAG-CGG-AC-3'; TGFB1-F forward, 5'-ACT-GCG-GAT-CTC-TGT-GTC-ATT-G-3' and reverse, 5'-ACA-GTA-GTG-TTC-CCC-ACT-GGT-C-3'; Vimentin forward, 5'-GGC-TCG-TCA-CCT-TCG-TGA-AT-3' and reverse, 5'-GAG-AAA-TCC-TGC-TCT-CCT-CGC-3'; $\beta$-actin forward, 5'-TTG-CTG-ACA-GGA-TGC-AGA-AG-3' and reverse, 5'-TGA-TCC-ACA-TCT-GCT-GGA-AG-3'). $\beta$-actin was used as an endogenous control to normalize the gene expression levels.

The expression of miRNAs was also carried out by RT-qPCR using the miScript SYBR ${ }^{\circledR}$-Green PCR kit (Qiagen, Inc.). Total cellular RNA extraction and quantification was performed as indicated above, whereas cDNA synthesis was executed with the miScript II RT kit (Qiagen, Inc.). Hsa-miR-21-5p (miR-21, 5'-UAGCUUAUCAGACUGAUG UUGA-3') was designed and used during this experiment and SNORD6 small nucleolar RNA, C/D box 6 (also known 

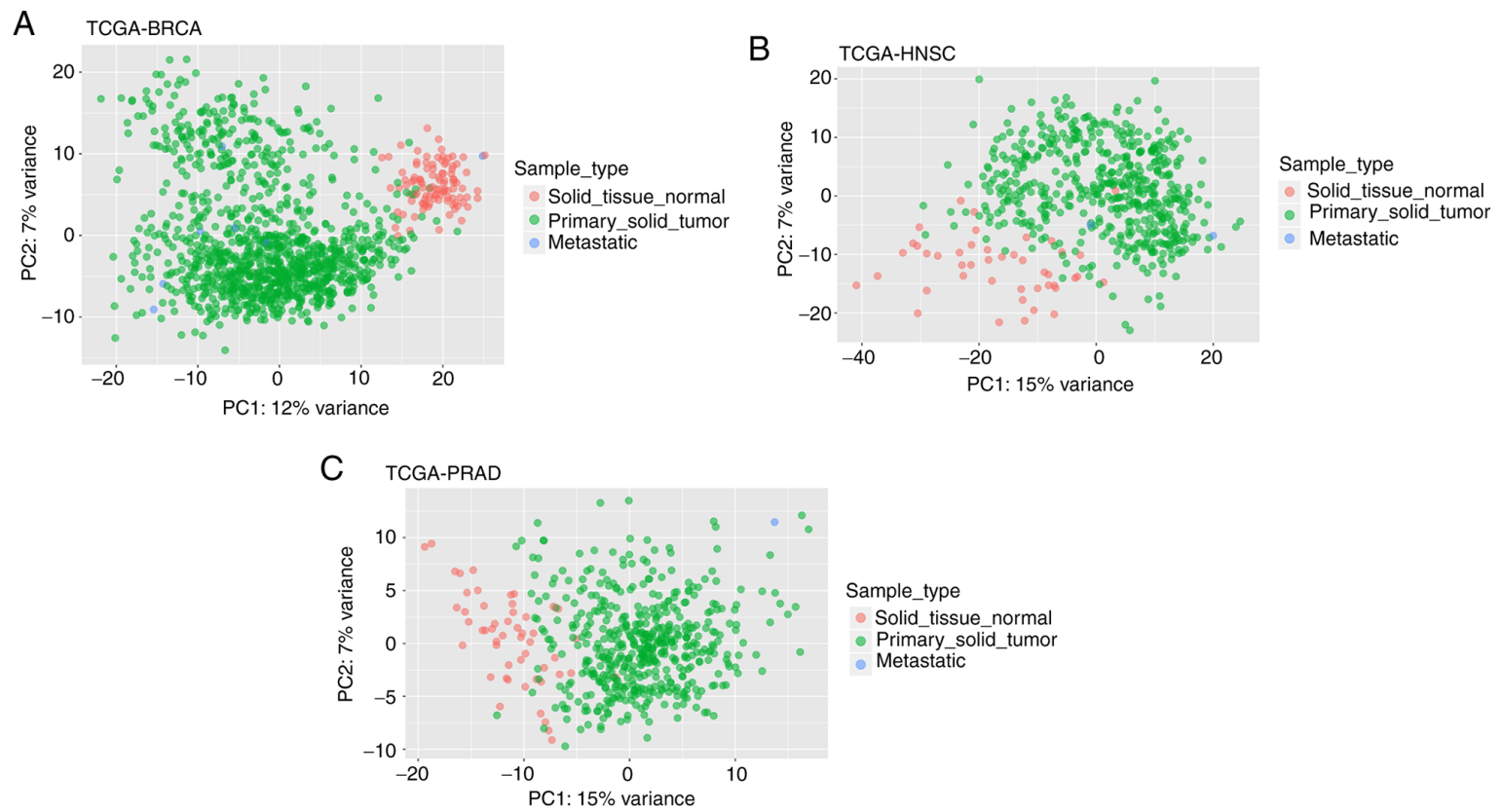

Figure 2. PCA of patient samples from (A) breast cancer, (B) head and neck cancer and (C) prostate cancer using the expression values of differentially expressed miRNAs (please see the 'Materials and methods' section). PCA was performed and scatterplots were created using DESeq2 (13). differentially expressed genes. PCA was performed and scatterplots were created using DESeq2 (13). PCA, principal components analysis; TCGA, The Cancer Genome Atlas; BRCA, breast cancer; HNSC, head and neck squamous cell carcinoma; PRAD, prostate adenocarcinoma.

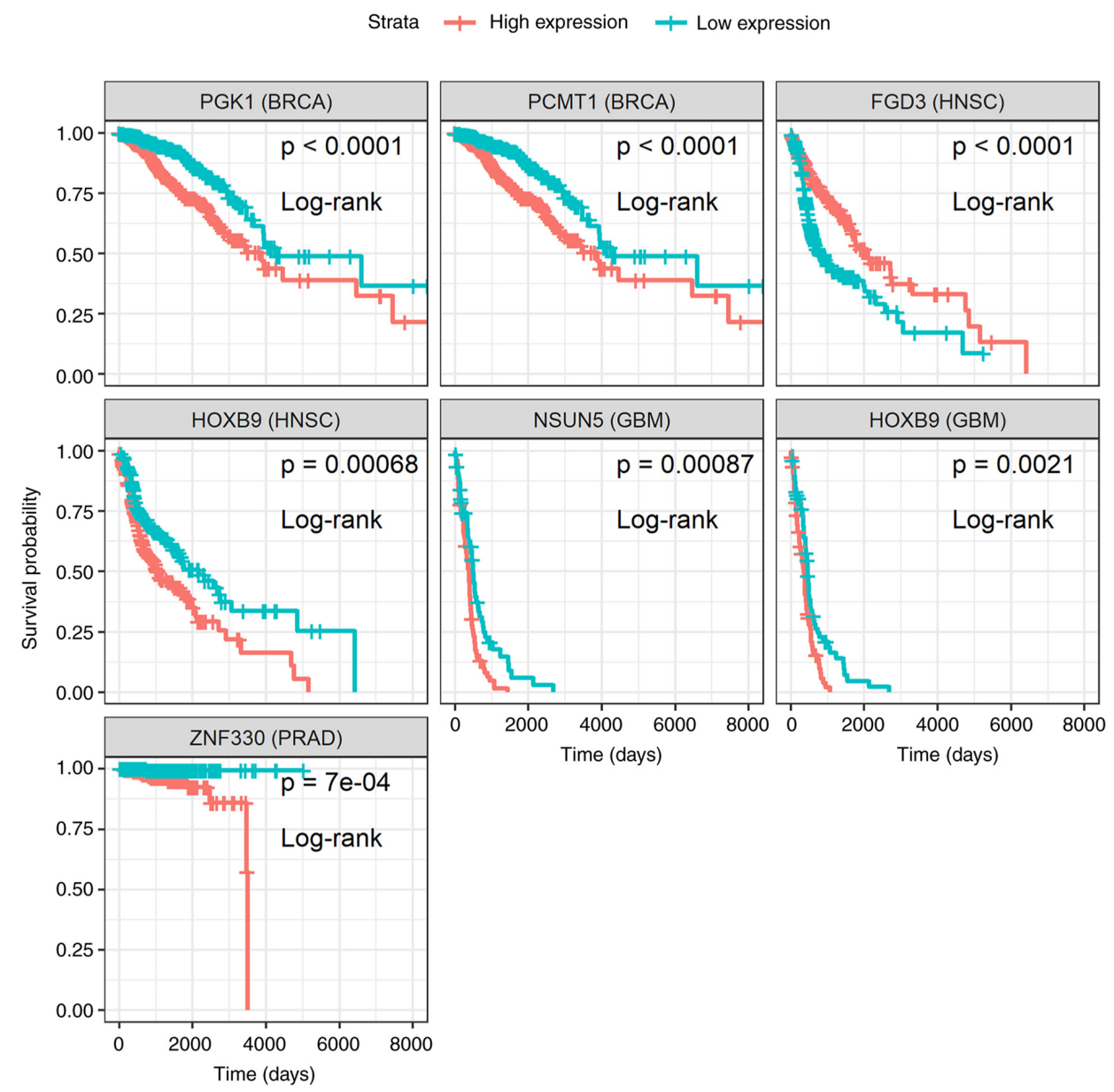

Figure 3. Kaplan-Meier survival curves of the differentially expressed genes, $P G K 1, P C M T 1, F G D 3, H O X B 9, N S U N 5$ and ZNF330, whose expression levels were significantly associated (P-value $<0.001$; non-parametric log-rank test) with a favorable or poor survival probability of patients with breast (BRCA), head and neck (HNSC), glioblastoma (GBM) or prostate (PRAD) cancer. PST samples were assigned into two separate groups depending on whether target expression of each sample is higher (high expression) or lower (low expression) than the median. Survival analysis was performed and plots were created using survival methods $(16,17)$ and survminer $(18)$. PST, primary solid tumor; (Time: Is shown in days). 


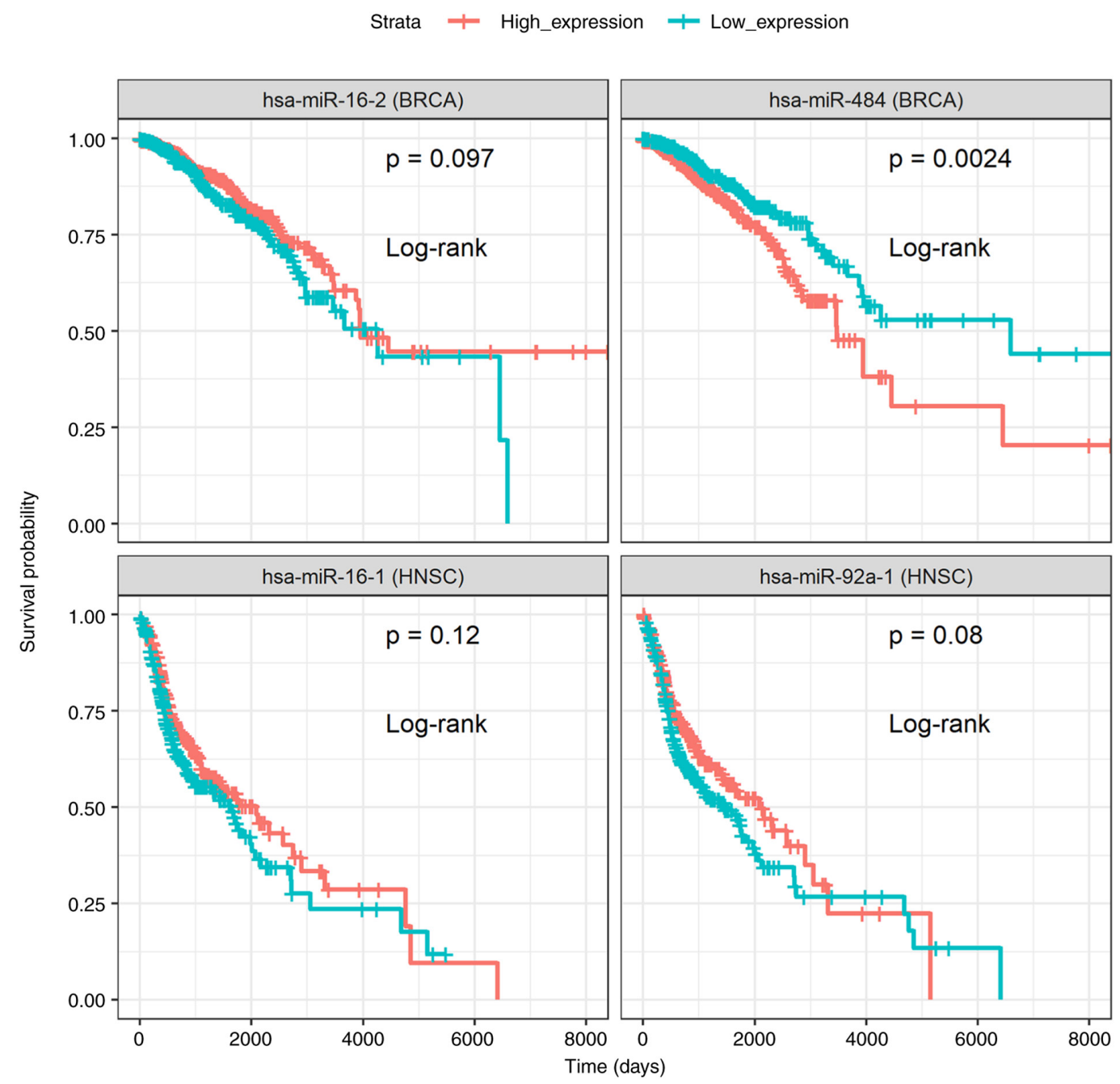

Figure 4. Kaplan-Meier survival curves of differentially expressed miRNAs miR-16-1/-2, miR-92-1 and miR-484, whose expression levels are significantly associated (P-value $~ 0.1$; non-parametric log-rank test) with favorable or poor survival probability of patients with breast (BRCA) or head and neck (HNSC) cancer. PST samples were assigned into two separate groups depending on whether target expression of each sample is higher (high expression) or lower (low expression) than the median. Survival analysis was performed and plots were created using survival methods $(16,17)$ and survminer $(18)$. PST, primary solid tumor; (Time: Is shown in days).

as mgh28S-2412) (Qiagen, Inc.) was used as reference RNA gene. The reaction conditions consisted of polymerase activation/denaturation at $95^{\circ} \mathrm{C}$ for $15 \mathrm{~min}$, followed by 40 cycles at $94^{\circ} \mathrm{C}$ for $15 \mathrm{sec}$ and $55^{\circ} \mathrm{C}$ for $30 \mathrm{sec}$.

In both cases, the relative $\mathrm{mRNA} / \mathrm{miRNA}$ concentrations were calculated using the $2^{-\Delta \Delta \mathrm{Cq}}$ method (21) and the results obtained were represented as fold changes in the diagrams.

Statistical analysis. The results from 2 independent biological experiments (triplicate measurements) are shown and the data are expressed as the means \pm standard deviation (SE). Comparisons were carried out using a Student's t test, whereas the statistical analysis was performed using GraphPad Prism 6.0 (GraphPad Software, Inc.). P<0.05 was considered to indicate a statistically significant difference.

\section{Results}

DE genes and miRNAs. Accessing the GDC data portal through TCGAbiolinks enabled the retrieval of RNA-Seq for 4 different types of malignancies. For each project, STN and
PST were identified as the predominant categories containing the majority of samples. Specifically, for TCGA-BRCA, TCGA-HNSC, TCGA-PRAD and TCGA-GBM, STN + PST samples numbering $113+1,102,44+523,52+498$ and $5+156$, respectively, were obtained, filtered for low count genes and compared to identify DE genes. Based on strict criteria (please see the 'Materials and methods' section) a subset of all genes analyzed was determined to be DE genes in each tumor (percentage of DE genes in each TCGA-project: TCGA-BRCA, 8.95\%; TCGA-HNSC, 6.1\%; TCGA-PRAD, 2.56\%; TCGA-GBM, $6.52 \%$ ) (Table I). Similarly, miRNA-Seq data were retrieved for 3 TCGA projects and major sample categories STN and PST were used for DE miRNA identification (TCGA-BRCA, TCGA-HNSC and TCGA-PRAD with STN + PST samples numbering $104+1,096,44+523$, $52+498$, respectively). Following analysis, the percentage of DE miRNAs were determined to be as follows: TCGA-BRCA, 19.1\%; TCGA-HNSC, 18.33\%; TCGA-PRAD, 9.04\% (Table I).

To further confirm these findings, PCA analysis was performed using DE gene and miRNA expression values for all samples of each TCGA-project. Following dimension 
A

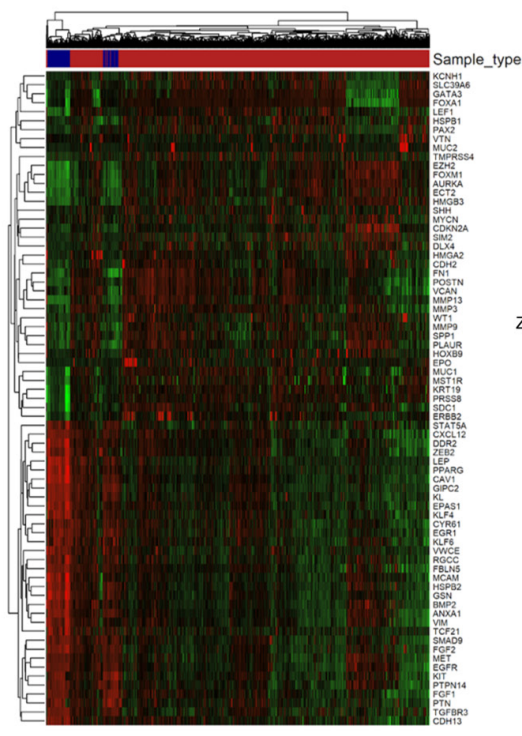

C

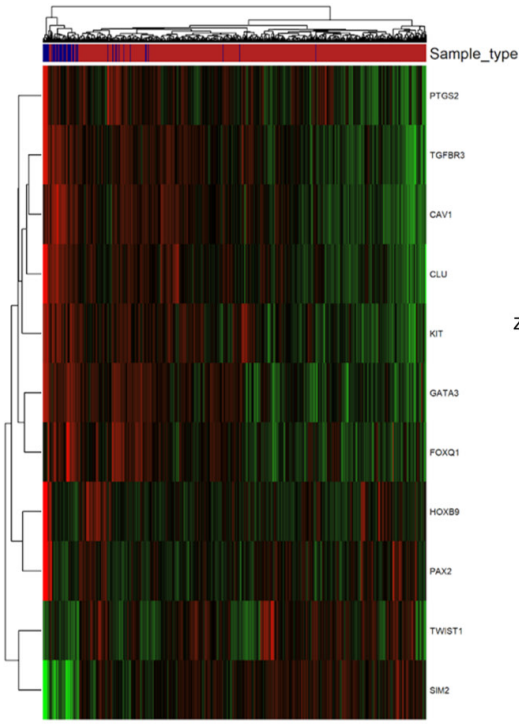

B

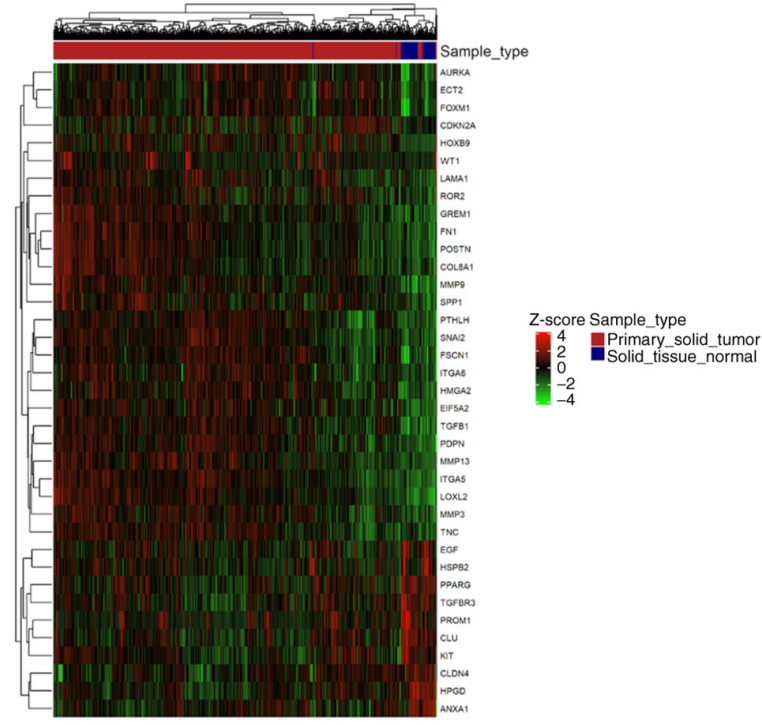

D

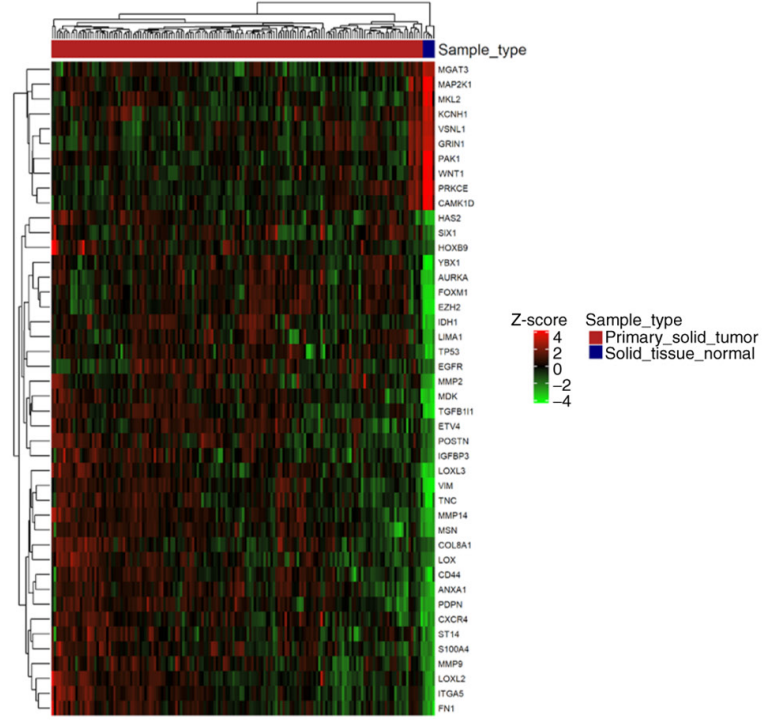

Figure 5. Heatmaps of expression for genes that are differentially expressed and EMT-associated in at least one of the following malignancies: (A) breast cancer, (B) head and neck cancer, (C) prostate cancer and/or (D) glioblastoma. Gene counts were transformed using variance-stabilizing transformation and scaled to z-score for each gene. Heatmaps were created using ComplexHeatmap (66). The number of genes reported for each malignancy heatmap, is available in Table I (differentially expressed genes reported by dbEMT 1.0). Gene names for each malignancy heatmap are also given in Table II (marked as 'TRUE' for each malignancy). EMT, epithelial-to-mesenchymal transition.

reduction, loadings of principal components (PC) 1 and 2 were plotted against each other and displayed the formation of distinct groups between STN and PST, as well as other sample types, such as metastatic, in each type of cancer (Figs. 1 and 2).

Moreover, survival analysis was performed on DE genes and miRNAs (please see the 'Materials and methods' section). In total, 6 genes (Fig. 3) and 3 miRNAs (Fig. 4) were reported, for which patients with high or low expression levels presented considerable differences in survival. The results concerning the association of the genes $P G K 1$, PCMT1, FDG3, HOXB9, NSUN5 and ZNF330 to patient survival probability, are in agreement with those previously reported by the Human Protein Atlas project (22), with the exception of the unfavorable prognosis of HOXB9 overexpression in glioblastoma, which was identified during the current analysis (Fig. 3). As regards the miRNAs,
miR-16, known for its tumor suppressive functions (23-25), miR-92a-1 (26) and miR-484 (27-29) exhibited an associated with a favorable and poor prognosis, respectively, for patients with breast and head and neck cancer (Fig. 4). These results support the approach for the identification of DE targets.

EMT-associated genes and miRNAs. In total, 344 genes and 20 miRNAs that, following an exhaustive literature search, constitute a collection of well-characterized EMT-associated targets, were retrieved from dbEMT 1.0 (10). Direct comparisons between the 2 gene collections revealed that only a small fraction of DE genes has been identified as directly related to the EMT process (percentage of DE genes that were EMT-related: TCGA-BRCA, 1.7\%; TCGA-HNSC, 1.5\%; TCGA-PRAD, 1\%; TCGA-GBM, 1.9\%) (Table I). A 
A

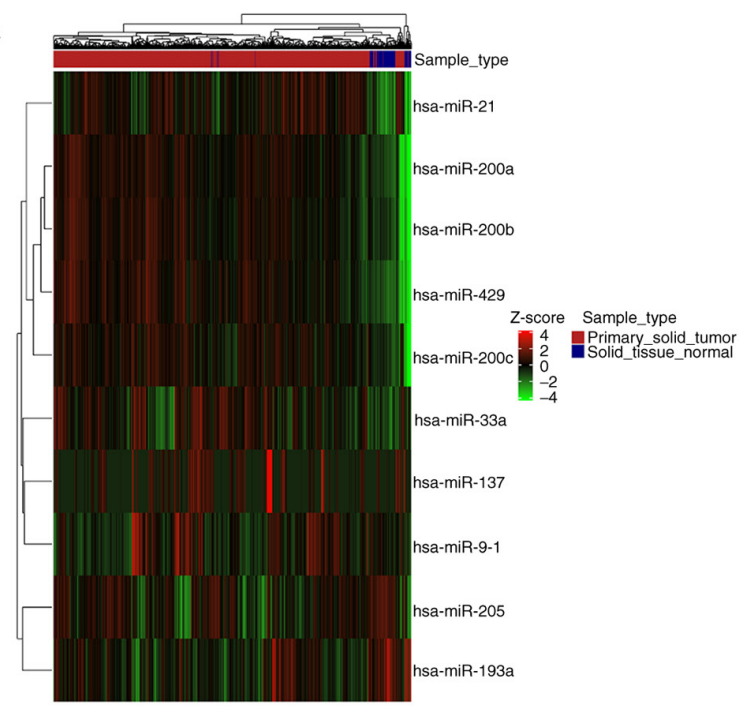

B

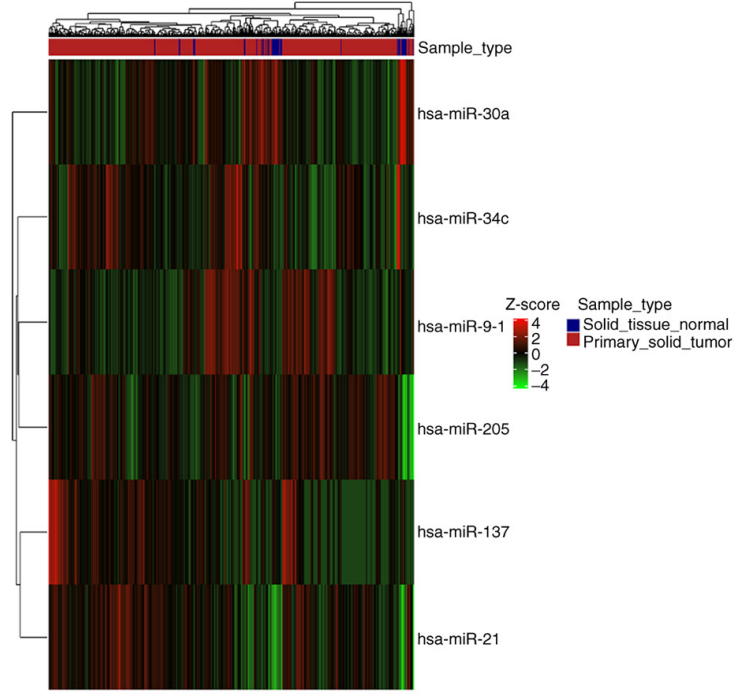

C

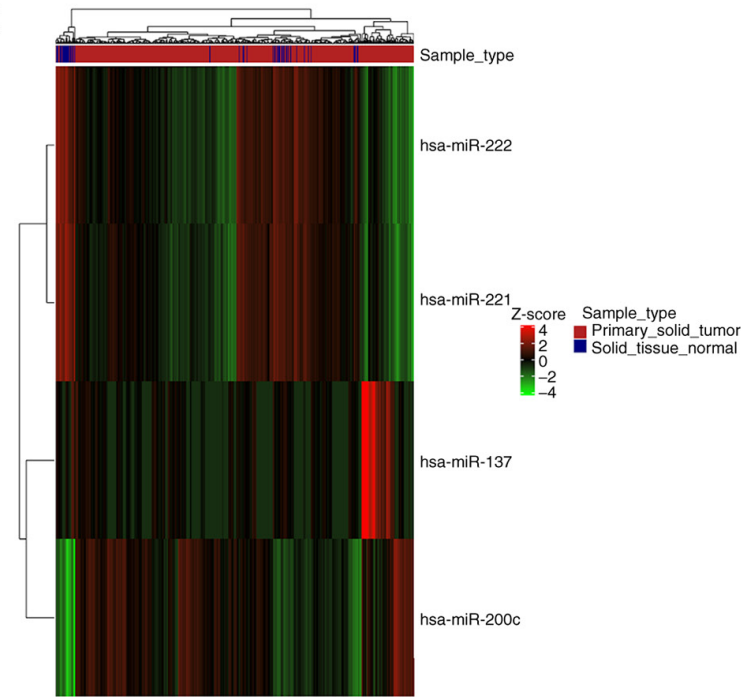

Figure 6. Heatmaps of expression for miRNAs that are differentially expressed and EMT-associated in at least one of the following malignancies: (A) breast cancer, (B) head and neck cancer and/or (C) prostate cancer. miRNA counts were transformed using variance-stabilizing transformation and scaled to z-score for each miRNA. Heatmaps were created using ComplexHeatmap (66). The number of miRNAs reported for each malignancy heatmap, is available in Table I (differentially expressed miRNAs reported by dbEMT 1.0). miRNA names for each malignancy heatmap are also given in Table III (marked as 'TRUE' for each malignancy). EMT, epithelial-to-mesenchymal transition.

small number of miRNAs reported in dbEMT 1.0 was also found in the present collection of DE miRNAs (percentage of DE miRNAs that were EMT-related: TCGA-BRCA, 3.7\%; TCGA-HNSC, 2.4\%; TCGA-PRAD, 3.6\%) (Table I).

Furthermore, DE genes and miRNAs related to EMT were found both up- and downregulated in each type of cancer (Figs. 5 and 6). Careful inspection of these results is required to decipher the role of each gene and miRNA in EMT, at the context of each malignancy.

EMT targets amongst different malignancies. With an aim of identifying targets that are commonly deregulated and EMT-associated between different types of cancer, pairwise comparisons of DE genes and miRNAs associated with EMT were performed. It was found that different types of malignancies shared several deregulated molecules (Tables II and III), with $H O X B 9$ and miR-137 being common for all types of malignancies that were examined (Fig. 7).
Assessment of DE genes and miRNAs related to EMT in breast, and head and neck cell carcinoma lines. Based on the data obtained, the present study wished to assess the expression level in a number of DE genes in well-characterized human breast, and head and neck cancer cell lines. To this end, the selection was made for caveolin-1, FOXM1 and Vimentin for the human breast epithelial carcinoma cell lines, MCF-7 and MDA-MB-231. As for the head and neck cancer cell lines, the human oral HSC-3 and keratinocyte HaCaT cancer cells were used to assess the gene expression levels of HMGA2, TGFB1, FOXM1 and PPARG. Moreover, from the DE miRNAs, the expression of miR-21 was selected and was assessed in all these 4 cell lines.

As shown in Fig. 8, for the breast epithelial carcinoma cell lines, the expression of caveolin-1 (Fig. 8A) was markedly higher in the MDA-MB-231 cells than in the MCF-7 cells $(\mathrm{P}<0.001)$. As regards the expression of miR-21 (Fig. 8B), it was higher again in the MDA-MB-231 cells compared to the MCF-7 cells, 
Table II. Differentially expressed and EMT-associated genes in different malignancies.

\begin{tabular}{|c|c|c|c|c|c|}
\hline Gene symbol & Ensemble gene ID & TCGA_BRCA & TCGA_HNSC & TCGA_PRAD & TCGA_GBM \\
\hline ZEB2 & ENSG00000169554 & TRUE & FALSE & FALSE & FALSE \\
\hline EGFR & ENSG00000146648 & TRUE & FALSE & FALSE & TRUE \\
\hline EPAS1 & ENSG00000116016 & TRUE & FALSE & FALSE & FALSE \\
\hline ERBB2 & ENSG00000141736 & TRUE & FALSE & FALSE & FALSE \\
\hline MET & ENSG00000105976 & TRUE & FALSE & FALSE & FALSE \\
\hline $\mathrm{CDH} 2$ & ENSG00000170558 & TRUE & FALSE & FALSE & FALSE \\
\hline KLF4 & ENSG00000136826 & TRUE & FALSE & FALSE & FALSE \\
\hline KLF6 & ENSG00000067082 & TRUE & FALSE & FALSE & FALSE \\
\hline WT1 & ENSG00000184937 & TRUE & TRUE & FALSE & FALSE \\
\hline LEF1 & ENSG00000138795 & TRUE & FALSE & FALSE & FALSE \\
\hline SIM2 & ENSG00000159263 & TRUE & FALSE & TRUE & FALSE \\
\hline FN1 & ENSG00000115414 & TRUE & TRUE & FALSE & TRUE \\
\hline EGR1 & ENSG00000120738 & TRUE & FALSE & FALSE & FALSE \\
\hline KIT & ENSG00000157404 & TRUE & TRUE & TRUE & FALSE \\
\hline BMP2 & ENSG00000125845 & TRUE & FALSE & FALSE & FALSE \\
\hline CAV1 & ENSG00000105974 & TRUE & FALSE & TRUE & FALSE \\
\hline PPARG & ENSG00000132170 & TRUE & TRUE & FALSE & FALSE \\
\hline TGFBR3 & ENSG00000069702 & TRUE & TRUE & TRUE & FALSE \\
\hline FOXA1 & ENSG00000129514 & TRUE & FALSE & FALSE & FALSE \\
\hline STAT5A & ENSG00000126561 & TRUE & FALSE & FALSE & FALSE \\
\hline GATA3 & ENSG00000107485 & TRUE & FALSE & TRUE & FALSE \\
\hline ANXA1 & ENSG00000135046 & TRUE & TRUE & FALSE & TRUE \\
\hline DDR2 & ENSG00000162733 & TRUE & FALSE & FALSE & FALSE \\
\hline FOXM1 & ENSG00000111206 & TRUE & TRUE & FALSE & TRUE \\
\hline HSPB1 & ENSG00000106211 & TRUE & FALSE & FALSE & FALSE \\
\hline VIM & ENSG00000026025 & TRUE & FALSE & FALSE & TRUE \\
\hline SMAD9 & ENSG00000120693 & TRUE & FALSE & FALSE & FALSE \\
\hline GSN & ENSG00000148180 & TRUE & FALSE & FALSE & FALSE \\
\hline CYR61 & ENSG00000142871 & TRUE & FALSE & FALSE & FALSE \\
\hline MST1R & ENSG00000164078 & TRUE & FALSE & FALSE & FALSE \\
\hline VCAN & ENSG00000038427 & TRUE & FALSE & FALSE & FALSE \\
\hline MYCN & ENSG00000134323 & TRUE & FALSE & FALSE & FALSE \\
\hline TCF21 & ENSG00000118526 & TRUE & FALSE & FALSE & FALSE \\
\hline HMGA2 & ENSG00000149948 & TRUE & TRUE & FALSE & FALSE \\
\hline CDKN2A & ENSG00000147889 & TRUE & TRUE & FALSE & FALSE \\
\hline MUC1 & ENSG00000185499 & TRUE & FALSE & FALSE & FALSE \\
\hline AURKA & ENSG00000087586 & TRUE & TRUE & FALSE & TRUE \\
\hline FGF2 & ENSG00000138685 & TRUE & FALSE & FALSE & FALSE \\
\hline MCAM & ENSG00000076706 & TRUE & FALSE & FALSE & FALSE \\
\hline PAX2 & ENSG00000075891 & TRUE & FALSE & TRUE & FALSE \\
\hline PTPN14 & ENSG00000152104 & TRUE & FALSE & FALSE & FALSE \\
\hline $\mathrm{SHH}$ & ENSG00000164690 & TRUE & FALSE & FALSE & FALSE \\
\hline SPP1 & ENSG00000118785 & TRUE & TRUE & FALSE & FALSE \\
\hline CDH13 & ENSG00000140945 & TRUE & FALSE & FALSE & FALSE \\
\hline VTN & ENSG00000109072 & TRUE & FALSE & FALSE & FALSE \\
\hline FBLN5 & ENSG00000140092 & TRUE & FALSE & FALSE & FALSE \\
\hline KRT19 & ENSG00000171345 & TRUE & FALSE & FALSE & FALSE \\
\hline $\mathrm{EZH} 2$ & ENSG00000106462 & TRUE & FALSE & FALSE & TRUE \\
\hline ECT2 & ENSG00000114346 & TRUE & TRUE & FALSE & FALSE \\
\hline PLAUR & ENSG00000011422 & TRUE & FALSE & FALSE & FALSE \\
\hline MMP9 & ENSG00000100985 & TRUE & TRUE & FALSE & TRUE \\
\hline PTN & ENSG00000105894 & TRUE & FALSE & FALSE & FALSE \\
\hline
\end{tabular}


Table II. Continued.

\begin{tabular}{|c|c|c|c|c|c|}
\hline Gene symbol & Ensemble gene ID & TCGA_BRCA & TCGA_HNSC & TCGA_PRAD & TCGA_GBM \\
\hline POSTN & ENSG00000133110 & TRUE & TRUE & FALSE & TRUE \\
\hline CXCL12 & ENSG00000107562 & TRUE & FALSE & FALSE & FALSE \\
\hline EPO & ENSG00000130427 & TRUE & FALSE & FALSE & FALSE \\
\hline FGF1 & ENSG00000113578 & TRUE & FALSE & FALSE & FALSE \\
\hline DLX4 & ENSG00000108813 & TRUE & FALSE & FALSE & FALSE \\
\hline MMP3 & ENSG00000149968 & TRUE & TRUE & FALSE & FALSE \\
\hline LEP & ENSG00000174697 & TRUE & FALSE & FALSE & FALSE \\
\hline PRSS8 & ENSG00000052344 & TRUE & FALSE & FALSE & FALSE \\
\hline MMP13 & ENSG00000137745 & TRUE & TRUE & FALSE & FALSE \\
\hline KL & ENSG00000133116 & TRUE & FALSE & FALSE & FALSE \\
\hline HOXB9 & ENSG00000170689 & TRUE & TRUE & TRUE & TRUE \\
\hline SLC39A6 & ENSG00000141424 & TRUE & FALSE & FALSE & FALSE \\
\hline SDC1 & ENSG00000115884 & TRUE & FALSE & FALSE & FALSE \\
\hline GIPC2 & ENSG00000137960 & TRUE & FALSE & FALSE & FALSE \\
\hline HMGB3 & ENSG00000029993 & TRUE & FALSE & FALSE & FALSE \\
\hline TMPRSS4 & ENSG00000137648 & TRUE & FALSE & FALSE & FALSE \\
\hline RGCC & ENSG00000102760 & TRUE & FALSE & FALSE & FALSE \\
\hline VWCE & ENSG00000167992 & TRUE & FALSE & FALSE & FALSE \\
\hline MUC2 & ENSG00000198788 & TRUE & FALSE & FALSE & FALSE \\
\hline $\mathrm{KCNH} 1$ & ENSG00000143473 & TRUE & FALSE & FALSE & TRUE \\
\hline HSPB2 & ENSG00000170276 & TRUE & TRUE & FALSE & FALSE \\
\hline TGFB1 & ENSG00000105329 & FALSE & TRUE & FALSE & FALSE \\
\hline SNAI2 & ENSG00000019549 & FALSE & TRUE & FALSE & FALSE \\
\hline EGF & ENSG00000138798 & FALSE & TRUE & FALSE & FALSE \\
\hline $\mathrm{TNC}$ & ENSG00000041982 & FALSE & TRUE & FALSE & TRUE \\
\hline ITGA6 & ENSG00000091409 & FALSE & TRUE & FALSE & FALSE \\
\hline PTHLH & ENSG00000087494 & FALSE & TRUE & FALSE & FALSE \\
\hline ITGA5 & ENSG00000161638 & FALSE & TRUE & FALSE & TRUE \\
\hline ROR2 & ENSG00000169071 & FALSE & TRUE & FALSE & FALSE \\
\hline CLDN4 & ENSG00000189143 & FALSE & TRUE & FALSE & FALSE \\
\hline HPGD & ENSG00000164120 & FALSE & TRUE & FALSE & FALSE \\
\hline GREM1 & ENSG00000166923 & FALSE & TRUE & FALSE & FALSE \\
\hline CLU & ENSG00000120885 & FALSE & TRUE & TRUE & FALSE \\
\hline LAMA1 & ENSG00000101680 & FALSE & TRUE & FALSE & FALSE \\
\hline PROM1 & ENSG00000007062 & FALSE & TRUE & FALSE & FALSE \\
\hline LOXL2 & ENSG00000134013 & FALSE & TRUE & FALSE & TRUE \\
\hline FSCN1 & ENSG00000075618 & FALSE & TRUE & FALSE & FALSE \\
\hline COL8A1 & ENSG00000144810 & FALSE & TRUE & FALSE & TRUE \\
\hline EIF5A2 & ENSG00000163577 & FALSE & TRUE & FALSE & FALSE \\
\hline PDPN & ENSG00000162493 & FALSE & TRUE & FALSE & TRUE \\
\hline TWIST1 & ENSG00000122691 & FALSE & FALSE & TRUE & FALSE \\
\hline PTGS2 & ENSG00000073756 & FALSE & FALSE & TRUE & FALSE \\
\hline FOXQ1 & ENSG00000164379 & FALSE & FALSE & TRUE & FALSE \\
\hline TP53 & ENSG00000141510 & FALSE & FALSE & FALSE & TRUE \\
\hline MAP2K1 & ENSG00000169032 & FALSE & FALSE & FALSE & TRUE \\
\hline PRKCE & ENSG00000171132 & FALSE & FALSE & FALSE & TRUE \\
\hline CD44 & ENSG00000026508 & FALSE & FALSE & FALSE & TRUE \\
\hline MMP2 & ENSG00000087245 & FALSE & FALSE & FALSE & TRUE \\
\hline YBX1 & ENSG00000065978 & FALSE & FALSE & FALSE & TRUE \\
\hline TGFB1I1 & ENSG00000140682 & FALSE & FALSE & FALSE & TRUE \\
\hline CXCR4 & ENSG00000121966 & FALSE & FALSE & FALSE & TRUE \\
\hline MDK & ENSG00000110492 & FALSE & FALSE & FALSE & TRUE \\
\hline
\end{tabular}


Table II. Continued.

\begin{tabular}{llcccc}
\hline Gene symbol & Ensemble gene ID & TCGA_BRCA & TCGA_HNSC & TCGA_PRAD & TCGA_GBM \\
\hline MSN & ENSG00000147065 & FALSE & FALSE & FALSE & TRUE \\
SIX1 & ENSG00000126778 & FALSE & FALSE & FALSE & TRUE \\
S100A4 & ENSG00000196154 & FALSE & FALSE & FALSE & TRUE \\
PAK1 & ENSG00000149269 & FALSE & FALSE & FALSE & TRUE \\
IGFBP3 & ENSG00000146674 & FALSE & FALSE & FALSE & TRUE \\
MMP14 & ENSG00000157227 & FALSE & FALSE & FALSE & TRUE \\
ST14 & ENSG00000149418 & FALSE & FALSE & FALSE & TRUE \\
MKL2 & ENSG00000186260 & FALSE & FALSE & FALSE & TRUE \\
ETV4 & ENSG00000175832 & FALSE & FALSE & FALSE & TRUE \\
WNT1 & ENSG00000125084 & FALSE & FALSE & FALSE & TRUE \\
LOX & ENSG00000113083 & FALSE & FALSE & FALSE & TRUE \\
LIMA1 & ENSG00000050405 & FALSE & FALSE & FALSE & TRUE \\
GRIN1 & ENSG00000176884 & FALSE & FALSE & FALSE & TRUE \\
LOXL3 & ENSG00000115318 & FALSE & FALSE & FALSE & TRUE \\
VSNL1 & ENSG00000163032 & FALSE & FALSE & FALSE & TRUE \\
IDH1 & ENSG00000138413 & FALSE & FALSE & FALSE & TRUE \\
CAMK1D & ENSG00000183049 & FALSE & FALSE & FALSE & TRUE \\
MGAT3 & ENSG00000128268 & FALSE & FALSE & FALSE & TRUE \\
HAS2 & ENSG00000170961 & FALSE & FALSE & FALSE & TRUE \\
\hline
\end{tabular}

EMT, epithelial-to-mesenchymal transition; The Cancer Genome Atlas; BRCA, breast cancer; HNSC, head and neck squamous cell carcinoma; PRAD, prostate adenocarcinoma; GBM, glioblastoma multiforme.

Table III. Differentially expressed and EMT-associated miRNAs in different malignancies.

\begin{tabular}{llll}
\hline miRNA & TCGA_BRCA & TCGA_HNSC & TCGA_PRAD \\
\hline hsa-mir-137 & TRUE & TRUE & TRUE \\
hsa-mir-193a & TRUE & FALSE & FALSE \\
hsa-mir-200a & TRUE & FALSE & FALSE \\
hsa-mir-200b & TRUE & FALSE & FALSE \\
hsa-mir-200c & TRUE & FALSE & TRUE \\
hsa-mir-205 & TRUE & TRUE & FALSE \\
hsa-mir-21 & TRUE & TRUE & FALSE \\
hsa-mir-33a & TRUE & FALSE & FALSE \\
hsa-mir-9-1 & TRUE & TRUE & FALSE \\
hsa-mir-429 & TRUE & FALSE & FALSE \\
hsa-mir-30a & FALSE & TRUE & FALSE \\
hsa-mir-34c & FALSE & TRUE & FALSE \\
hsa-mir-221 & FALSE & FALSE & TRUE \\
hsa-mir-222 & FALSE & FALSE & TRUE \\
\hline
\end{tabular}

EMT, epithelial-to-mesenchymal transition; The Cancer Genome Atlas; BRCA, breast cancer; HNSC, head and neck squamous cell carcinoma; PRAD, prostate adenocarcinoma; GBM, glioblastoma multiforme.

although the difference was not statistically significant. Such gene and miR-21 differential expression profiles may contribute to the observed metabolic and phenotypic behavior of these 2 cell lines. Indeed, although they are both invasive ductal breast carcinoma cells, they have a number of phenotypic and genotypic differences. MCF-7 are estrogen receptor-positive cells, whereas MDA-MB-231 cells are estrogen and progesterone receptor-negative; in addition, MCF-7 cells express the epithelial phenotype in contrast to the MDA-MB-231 cells that are more mesenchymal (30-32).

Similarly, as shown in Fig. 9, the expression profiles of the FOXM1 and HMGA2 genes exhibited higher levels in the HSC-3 as compared to the HaCaT cells (Fig. 9A), although this difference was not statistically significant. On the contrary, miR-21 was found to have an increased expression in HaCaT compared to HSC-3 cells, a result that was again, not significant. It is interesting that the pattern of expression of these EMT-related genes and that of miR-21 varies in these 2 cell lines; however, it is still not known to what extent such behavior contributes to any metabolic and phenotypic property seen in these 2 cell lines.

\section{Discussion}

Through preliminary in silico analysis, the present study identified a list of genes and miRNAs that were DE and associated with EMT and/or MET in different types of cancer, such as head and neck, breast and prostate cancer, and glioblastoma. Moreover, RT-qPCR analysis revealed differential expression profiles of selected EMT-related genes and miR-21 in a number of human breast, and head and neck carcinoma cell lines. TCGA-BRCA and -HNSC cancer samples shared the most DE and dbEMT 1.0 reported genes and miRNAs, in accordance with their common epithelial tissue origin. The present study, 
A

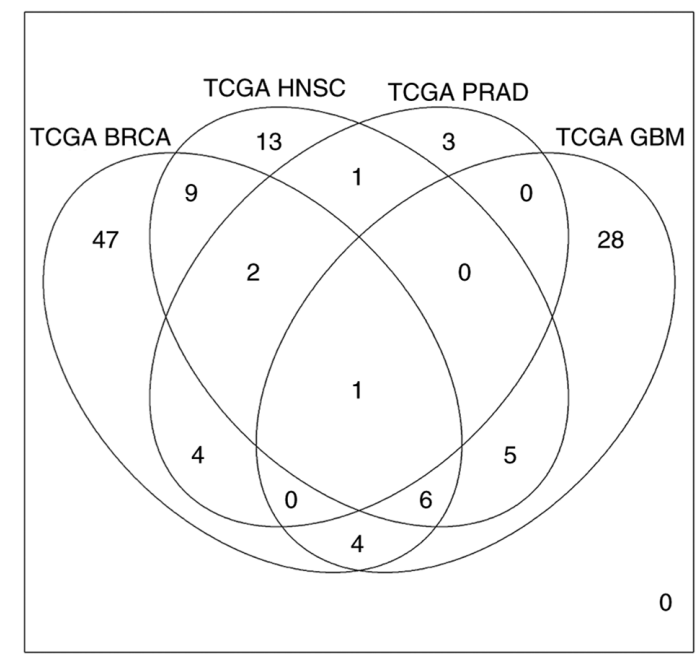

$\mathrm{B}$

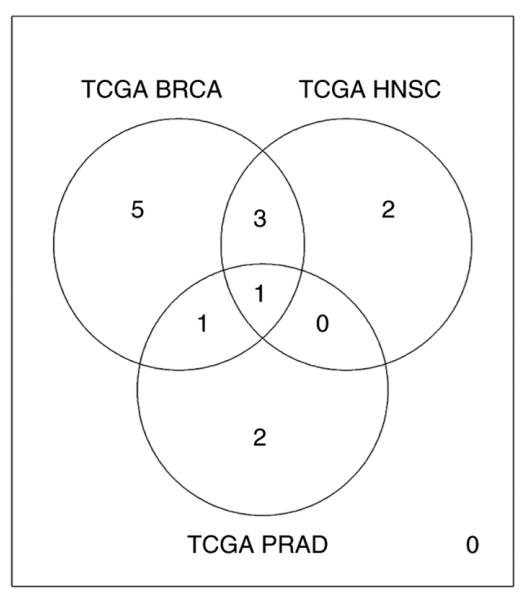

Figure 7. Venn diagrams showing common and unique differentially expressed and EMT reported (A) genes and (B) miRNAs for all types of malignancies that were analyzed. Plots were created using limma (15). EMT, epithelial-to-mesenchymal transition; The Cancer Genome Atlas; BRCA, breast cancer; HNSC, head and neck squamous cell carcinoma; PRAD, prostate adenocarcinoma; GBM, glioblastoma multiforme.

A

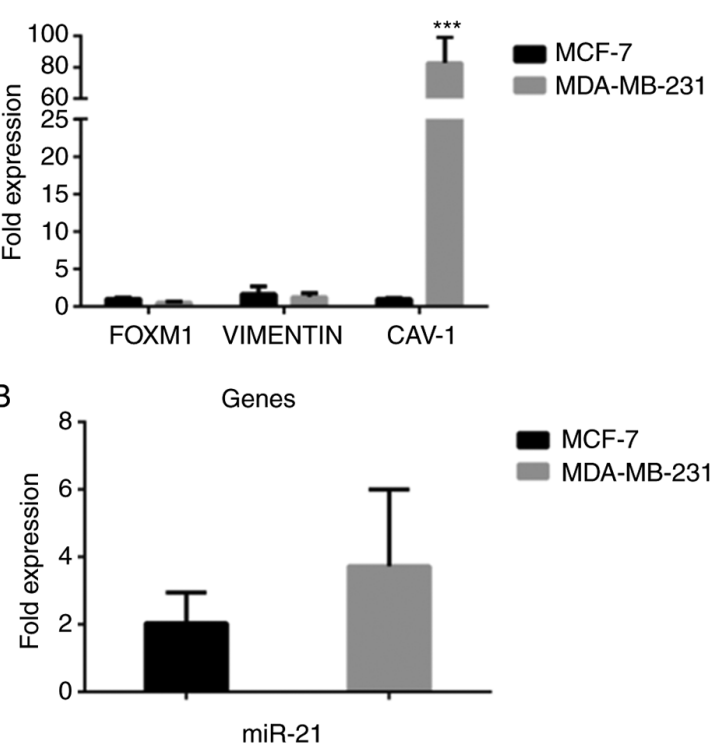

Figure 8. Assessment of expression profiles of (A) EMT-related genes and (B) miR-21 by RT-qPCR analysis of the MCF-7 and MDA-MB-231 cell lines. EMT, epithelial-to-mesenchymal transition. The results represent the means $\pm \mathrm{SD}(\mathrm{A}){ }^{* * *} \mathrm{P}<0.001$; (B) no statistically significant difference was observed; Student's t-test).

by analyzing the expression profiles of EMT-related genes and miRNAs in patient cancer samples, suggests that HOXB9 and miR-137 present the same deregulated patterns, independent of tumor type. HOXB9 belongs to HOX gene family that in human plays crucial role in physiology and pathophysiology, by modulating cell development, differentiation and growth. It is noteworthy that the aberrant expression of HOX genes has been shown to contribute to cancer progression and development $(33,34)$. Indeed, it has been observed that HOX genes exhibit a dysregulated expression in leukemia, ovarian and lung cancer (35-38). The function of HOXB9 novel tumor suppressor in the regulation of colon adenocarcinoma progression has also been identified (39). Moreover, it has been shown
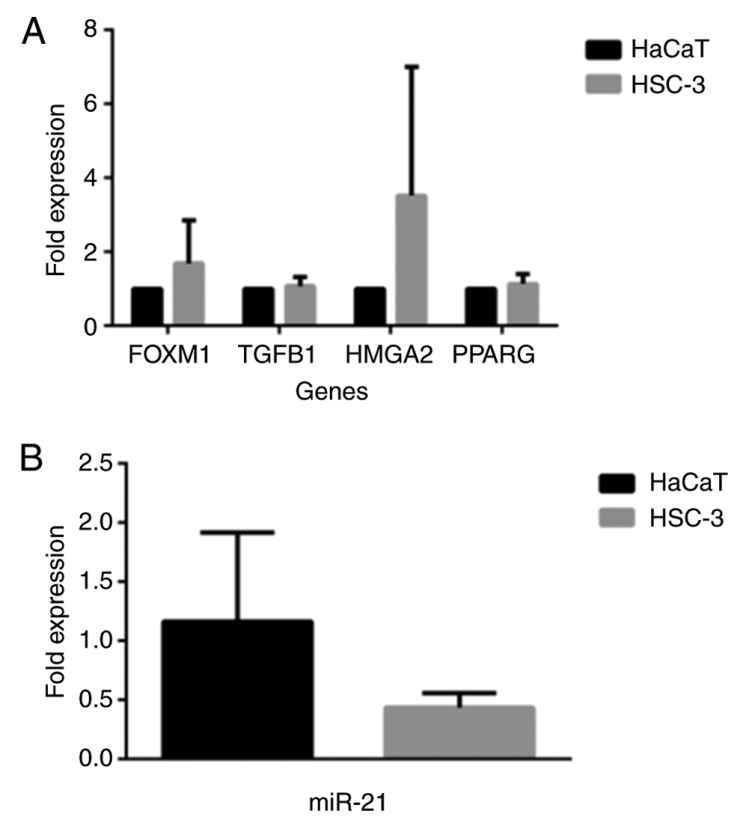

Figure 9. Assessment of expression profiles of (A) EMT-related genes and (B) miR-21 by RT-qPCR analysis of the HSC-3 and HaCaT cell lines. EMT, epithelial-to-mesenchymal transition. The results shown represent the mean \pm SD (no statistically significant difference is observed; Student's t-test).

that HOXB9 is associated with the emergence of radioresistance, as well as the development of resistance in anti-VEGF therapy in colorectal cancer $(40,41)$. Importantly, a recent study identified HOXB9 as one key gene in a 5-gene molecular prognostic signature in patients with laryngeal cancer (42). In addition, the implication of HOX9 in prostate cancer cell progression has been recently proposed (43). Furthermore, the present study reports HOXB9, along with PGK1 $(44,45)$, PCMT1 $(46,47)$, NSUN5 $(48,49)$ and ZNF330 $(50)$, as unfavorable markers for the survival of patients with the types of cancer examined herein. Of note, the present study demonstrated showed that HOXB9 overexpression was associated with a poor prognosis for patients with both head and neck 
cancer, previously reported in Human Protein Atlas (22), and those with glioblastoma. An exception was FGD3 $(51,52)$, with its overexpression being predictive of a favorable outcome for patients with head and neck cancer (Fig. 3).

miRNAs represent important players in the post-transcriptionally regulation of gene expression. In this manner, they affect signaling pathways and cellular processes with implication in cancer progression and development (53-56). miR-137 has shown to control tumorigenesis, invasion and metastasis in pancreatic neuroendocrine tumors (56). Moreover, miR-137 exhibits crucial developmental roles in neuronal differentiation (57). In addition, miR-137, by modulating SLC1A5-dependent glutamine uptake, is involved in the progression of head and neck squamous cell carcinoma $(58,59)$. The significant role of miR-137 in the progression, diagnosis and prognosis of hepatocellular carcinoma has also been documented (60). The therapeutic potential of targeting miR-137 in non-small cell lung cancer (NSCLC) has been recently proposed (61). By retrospectively analyzing tumor patient data, the task of identifying potential druggable genes and miRNAs related to the process of epithelial mesenchymal plasticity and exhibiting deregulated expression levels in different malignancies has been set forth. Moreover, the data presented in the present study justify the affordability of identifying common druggable cancer biomarkers applicable to various tumors, in order to proceed thereafter, through the pharmacological assessment, to the development of successful anticancer therapeutics. Of note, HOXB9 and miR-137 were found to be deregulated in all types of malignancies that were analyzed. However, both were expressed in very low levels compared to other genes and/or miRNAs. Therefore, careful examination is required upon attempting to further clinically validate their usefulness and therapeutic applicability, as several DE genes and miRNAs related to EMT are also shared by different types of cancer that were analyzed herein. To this end, the regulatory network of miRNAs in EMT plasticity has been previously evaluated in breast cancer (62). Moreover, the EMT regulatory network includes a number of EMT-related transcription factors (e.g., the Snail and Zeb family) and epigenetic collaborative regulators (e.g., miR-34 for Snail and miR-200s for Zeb). Such interplay drives the well-orchestrated epithelial-mesenchymal transcriptional program, thus mediating the downstream biological effects (6). In the present study, the bioinformatics analysis focused on EMT-related miRNAs and genes dysregulated in various origin tumor patient samples and thus the findings obtained highlight only such conclusions. Whether or not there exists any functional involvement between the miRNAs and the genes identified, needs to be experimentally validated. It is interesting, however, to note that recent data highlight the yet unexplored role of miRNAs as regulators of Hox genes in hematopoiesis, through the elucidation of the role of miR-708 as a novel regulator of the Hoxa9 program in leukemia myeloid cells (63).

Overall, the analysis approach, is further strengthening the previously published data regarding the modulation of EMT in tumors and proposes that targeted research efforts focused on identifying common biomarkers could provide effective anticancer drugs. The results obtained support the notion that as such, druggable biomarkers could be considered the HOXB9 gene and/or miR-137, irrespective of the cured tumor type, although further clinical and experimental studies are also needed. Importantly, however, such a direction is expected to provide valuable therapeutic interventions in malignancies by contributing toward overcoming the existed cellular and genomic heterogeneity (inter- and intra-tumoral) and the differential pharmacological response seen among patients. In particular, the data provided herein support the notion of identifying druggable biomarkers that impinge on the fundamental cancer cell traits that provide the needed advantageous capacity of tumor cell metabolism to abrogate the molecular balance, as well as the existing physiological restriction signals between differentiation, apoptosis and proliferation. This notion of the pan-cancer clinical intervention and the implementation of informed clinical decisions are based on the profiling of genomic signatures and molecular biomarkers in cancer patients; the origin and type of histology of the tumor have already begun to be left. Indeed, the development of therapeutics showing pan-cancer capabilities present a new revolutionary therapeutic era, an approach mentioned as 'tumor-agnostic therapies'. Complementary to this, the ability to identify and clinically validate cancer biomarkers working irrespectively of the tumor type, permits the implementation of personalized cancer therapy in the clinical setting. The already marketed anticancer drugs, pembrolizumab, larotrectinib and entrectinib, belong to the class of tumor-agnostic therapies, by successfully receiving approval and being clinical used in patients with various types of tumor bearing common molecular features (64). Furthermore, the ability to implement cancer therapy with pharmacogenomics-guided therapeutic decisions offers the needed precision in clinical practice for the practical utilization of molecular profiling and biomarkers, as well as the outcome improvement in patients (65).

\section{Acknowledgements}

The statistical methods used in the present study were reviewed by Angelis Eleftherios, Professor of Statistics and Information Systems, Head of the School of Informatics, Faculty of Sciences, Aristotle University of Thessaloniki, Greece.

\section{Funding}

The present study was funded in the context of the project 'Molecular signatures analysis of three-dimensional cell cultures and circulating tumor cells in the treatment of cancer' (MIS 5004622) under the call for proposals 'Supporting researchers with emphasis on new researchers' (EDULLL 34). The project was co-financed by Greece and the European Union (European Social Fund-ESF) by the Operational Programme Human Resources Development, Education and Lifelong Learning 2014-2020.

\section{Availability of data and materials}

All data generated or analyzed during this study are included in this published article or are available from the corresponding author on reasonable request.

\section{Authors' contributions}

KAK was involved in the acquisition of data, analysis and interpretation of data, drafting the article and providing final 
approval. MGA was involved in the interpretation of the data, cell culture experiments and providing final approval. LPNG was involved in the interpretation of the data, drafting the article and providing final approval. NGG was involved in the interpretation of the data, revising the article and providing final approval. ISV conceived and designed the study, drafting the article, critical revision and provided final approval.

\section{Ethics approval and consent to participate}

Not applicable.

\section{Patient consent for publication}

Not applicable.

\section{Competing interests}

NGG declares ownership of Biogenea Pharmaceuticals Ltd. The company had no role in the design or outcomes of the study. The remaining authors declare that they have no competing interests.

\section{References}

1. Bocci F, Gearhart-Serna L, Boareto M, Ribeiro M, Ben-Jacob E, Devi GR, Levine H, Onuchic JN and Jolly MK: Toward understanding cancer stem cell heterogeneity in the tumor microenvironment. Proc Natl Acad Sci USA 116: 148-157, 2019.

2. Vizirianakis IS, Miliotou AN, Mystridis GA, Andriotis EG, Andreadis II, Papadopoulou LC and Fatouros DG: Tackling pharmacological response heterogeneity by PBPK modeling to advance precision medicine productivity of nanotechnology and genomics therapeutics. Expert Rev Precis Med Drug Dev 4 139-151, 2019.

3. Brabletz T, Kalluri R, Nieto MA and Weinberg RA: EMT in cancer. Nat Rev Cancer 18: 128-134, 2018.

4. Roche J: The epithelial-to-mesenchymal transition in cancer. Cancers (Basel) 10: 52, 2018.

5. Culig Z: Epithelial mesenchymal transition and resistance in endocrine-related cancers. Biochim Biophys Acta Mol Cell Res 1866: 1368-1375, 2019.

6. Lu W and Kang Y: Epithelial-mesenchymal plasticity in cancer progression and metastasis. Dev Cell 49: 361-374, 2019.

7. Williams ED, Gao D, Redfern A and Thompson EW: Controversies around epithelial-mesenchymal plasticity in cancer metastasis. Nat Rev Cancer 19: 716-732, 2019.

8. Kyrodimou M, Andreadis D, Drougou A, Amanatiadou EP, Angelis L, Barbatis C, Epivatianos A and Vizirianakis IS: Desmoglein-3/ $\gamma$-catenin and E-cadherin $/ \beta$-catenin differentia expression in oral leukoplakia and squamous cell carcinoma. Clin Oral Investig 18: 199-210, 2014.

9. Cancer Genome Atlas Research Network, Weinstein JN, Collisson EA, Mills GB, Shaw KR, Ozenberger BA, Ellrott K, Shmulevich I, Sander C and Stuart JM: The cancer genome atlas pan-cancer analysis project. Nat Genet 45: 1113-1120, 2013.

10. Zhao M, Kong L, Liu Y and Qu H: dbEMT: An epithelial-mesenchymal transition associated gene resource. Sci Rep 5: 11459, 2015.

11. Colaprico A, Silva TC, Olsen C, Garofano L, Cava C, Garolini D, Sabedot TS, Malta TM, Pagnotta SM, Castiglioni I, et al: TCGAbiolinks: An R/Bioconductor package for integrative analysis of TCGA data. Nucleic Acids Res 44: e71, 2016.

12. Anders S, Pyl PT and Huber W: HTSeq-a Python framework to work with high-throughput sequencing data. Bioinformatics 31 : $166-169,2015$.

13. Love MI, Huber W and Anders S: Moderated estimation of fold change and dispersion for RNA-seq data with DESeq2. Genome Biol 15: 550, 2014

14. Shaffer JP: Multiple hypothesis testing. Annu Rev Psychol 46: $561-584,1995$.
15. Ritchie ME, Phipson B, Wu D, Hu Y, Law CW, Shi W and Smyth GK: limma powers differential expression analyses for RNA-sequencing and microarray studies. Nucleic Acids Res 43: e47, 2015 .

16. Therneau TM and Grambsch PM: Modeling survival data: Extending the Cox model. Springer-Verlag New York, 2000.

17. Therneau T: A package for survival analysis in $\mathrm{R}$. $\mathrm{R}$ package version 3.2-7, 2020.

18. Kassambara A, Kosinski M and Biecek P: survminer: Drawing survival curves using'ggplot2', 2017.

19. Tseligka ED, Rova A, Amanatiadou EP, Calabrese G, Tsibouklis J, Fatouros DG and Vizirianakis IS: Pharmacological development of target-specific delocalized lipophilic cation-functionalized carboranes for cancer therapy. Pharm Res 33: 1945-1958, 2016.

20. Akrivou MG, Demertzidou VP, Theodoroula NF, Chatzopoulou FM, Kyritsis KA, Grigoriadis N, Zografos AL and Vizirianakis IS: Uncovering the pharmacological response of novel sesquiterpene derivatives that differentially alter gene expression and modulate the cell cycle in cancer cells. Int J Oncol 53: 2167-2179, 2018.

21. Livak KJ and Schmittgen TD: Analysis of relative gene expression data using real-time quantitative PCR and the 2(-Delta Delta C(T)) method. Methods 25: 402-408, 2001.

22. Uhlen M, Zhang C, Lee S, Sjöstedt E, Fagerberg L, Bidkhori G, Benfeitas R, Arif M, Liu Z, Edfors F, et al: A pathology atlas of the human cancer transcriptome. Science 357: eaan2507, 2017.

23. Liang H, Fu Z, Jiang X, Wang N, Wang F, Wang X, Zhang S, Wang Y, Yan X, Guan WX, et al: miR-16 promotes the apoptosis of human cancer cells by targeting FEAT. BMC Cancer 15: 448, 2015.

24. Qu Y, Liu H, Lv X, Liu Y, Wang X, Zhang M, Zhang X, Li Y, Lou Q, Li S and Li H: MicroRNA-16-5p overexpression suppresses proliferation and invasion as well as triggers apoptosis by targeting VEGFA expression in breast carcinoma. Oncotarget 8: 72400-72410, 2017.

25. Ruan L and Qian X: MiR-16-5p inhibits breast cancer by reducing AKT3 to restrain NF- $\kappa$ B pathway. Biosci Rep 39: BSR20191611, 2019.

26. Nilsson S, Möller C, Jirström K, Lee A, Busch S, Lamb R and Landberg G: Downregulation of miR-92a is associated with aggressive breast cancer features and increased tumour macrophage infiltration. PLoS One 7: e36051, 2012.

27. Zearo S, Kim E, Zhu Y, Zhao JT, Sidhu SB, Robinson BG and Soon PS: MicroRNA-484 is more highly expressed in serum of early breast cancer patients compared to healthy volunteers. BMC Cancer 14: 200, 2014.

28. Volinia S and Croce CM: Prognostic microRNA/mRNA signature from the integrated analysis of patients with invasive breast cancer. Proc Natl Acad Sci USA 110: 7413-7417, 2013.

29. Ye FG, Song CG, Cao ZG, Xia C, Chen DN, Chen L, Li S, Qiao F, Ling H, Yao L, et al: Cytidine deaminase axis modulated by miR-484 differentially regulates cell proliferation and chemoresistance in breast cancer. Cancer Res 75: 1504-1515, 2015.

30. Thompson EW, Reich R, Shima TB, Albini A, Graf J, Martin GR, Dickson RB and Lippman ME: Differential regulation of growth and invasiveness of MCF-7 breast cancer cells by antiestrogens. Cancer Res 48: 6764-6768, 1988.

31. Gjerdrum C, Tiron C, Høiby T, Stefansson I, Haugen H, Sandal T, Collett K, Li S, McCormack E, Gjertsen BT, et al: Axl is an essential epithelial-to-mesenchymal transition-induced regulator of breast cancer metastasis and patient survival. Proc Natl Acad Sci USA 107: 1124-1129, 2010.

32. Theodossiou TA, Ali M, Grigalavicius M, Grallert B, Dillard P, Schink KO, Olsen CE, Wälchli S, Inderberg EM, Kubin A, et al: Simultaneous defeat of MCF7 and MDA-MB-231 resistances by a hypericin PDT-tamoxifen hybrid therapy. NPJ Breast Cancer 5: $13,2019$.

33. Sha L, Dong L, Lv L, Bai L and Ji X: HOXB9 promotes epithelial-to-mesenchymal transition via transforming growth factor- $\beta 1$ pathway in hepatocellular carcinoma cells. Clin Exp Med 15: 55-64, 2015.

34. Bhatlekar S, Fields JZ and Boman BM: Role of HOX genes in stem cell differentiation and cancer. Stem Cells Int 2018: 3569493, 2018.

35. Hayashida T, Takahashi F, Chiba N, Brachtel E, Takahashi M, Godin-Heymann N, Gross KW, Vivanco Md, Wijendran V, Shioda T, et al: HOXB9, a gene overexpressed in breast cancer, promotes tumorigenicity and lung metastasis. Proc Natl Acad Sci USA 107: 1100-1105, 2010 
36. De Braekeleer E, Douet-Guilbert N, Basinko A, Le Bris MJ, Morel F and De Braekeleer M: Hox gene dysregulation in acute myeloid leukemia. Futur Oncol 10: 475-495, 2014

37. Eoh KJ, Kim HJ, Lee JY, Nam EJ, Kim S, Kim SW and Kim YT: Dysregulated expression of homeobox family genes may influence survival outcomes of patients with epithelial ovarian cancer: Analysis of data from the cancer genome atlas. Oncotarget 8: 70579-70585, 2017.

38. Song J, Wang T, Xu W, Wang P, Wan J, Wang Y, Zhan J and Zhang H: HOXB9 acetylation at K27 is responsible for its suppression of colon cancer progression. Cancer Lett 426: 63-72, 2018.

39. Zhan J, Niu M, Wang P, Zhu X, Li S, Song J, He H, Wang Y, Xue L, Fang W and Zhang H: Elevated HOXB9 expression promotes differentiation and predicts a favourable outcome in colon adenocarcinoma patients. Br J Cancer 111: 883-893, 2014.

40. Chiba N, Comaills V, Shiotani B, Takahashi F, Shimada T, Tajima K, Winokur D, Hayashida T, Willers H, Brachtel E, et al: Homeobox B9 induces epithelial-to-mesenchymal transition-associated radioresistance by accelerating DNA damage responses. Proc Natl Acad Sci USA 109: 2760-2765, 2012.

41. Carbone C, Piro G, Simionato F, Ligorio F, Cremolini C, Loupakis F, Alì G, Rossini D, Merz V, Santoro R, et al: Homeobox B9 mediates resistance to anti-VEGF therapy in colorectal cancer patients. Clin Cancer Res 23: 4312-4322, 2017.

42. Zhang G, Fan E, Yue G, Zhong Q, Shuai Y, Wu M, Feng G, Chen $\mathrm{Q}$ and Gou X: Five genes as a novel signature for predicting the prognosis of patients with laryngeal cancer. J Cell Biochem, Oct 31, 2019 (Online ahead of print).

43. Xu H, Wu S, Shen X, Wu D, Qin Z, Wang H, Chen X and Sun X: Silencing of HOXB9 suppresses cellular proliferation, angiogenesis, migration and invasion of prostate cancer cells. J Biosci 45: 40, 2020.

44. Fu D, He C, Wei J, Zhang Z, Luo Y, Tan H and Ren C: PGK1 is a potential survival biomarker and invasion promoter by regulating the HIF-1 $\alpha$-mediated epithelial-mesenchymal transition process in breast cancer. Cell Physiol Biochem 51: 2434-2444, 2018.

45. He Y, Luo Y, Zhang D, Wang X, Zhang P, Li H, Ejaz S and Liang S: PGK1-mediated cancer progression and drug resistance. Am J Cancer Res 9: 2280-2302, 2019.

46. Sambri I, Capasso R, Pucci P, Perna AF and Ingrosso D: The microRNA 15a/16-1 cluster down-regulates protein repair isoaspartyl methyltransferase in hepatoma cells: Implications for apoptosis regulation. J Biol Chem 286: 43690-43700, 2011.

47. Dong L, Li Y, Xue D and Liu Y: PCMT1 is an unfavorable predictor and functions as an oncogene in bladder cancer. IUBMB Life 70: 291-299, 2018.

48. Schosserer M, Minois N, Angerer TB, Amring M, Dellago H, Harreither E, Calle-Perez A, Pircher A, Gerstl MP, Pfeifenberger S, et al: Methylation of ribosomal RNA by NSUN5 is a conserved mechanism modulating organismal lifespan. Nat Commun 6: 6158, 2015.

49. Heissenberger C, Liendl L, Nagelreiter F, Gonskikh Y, Yang G, Stelzer EM, Krammer TL, Micutkova L, Vogt S, Kreil DP, et al: Loss of the ribosomal RNA methyltransferase NSUN5 impairs global protein synthesis and normal growth. Nucleic Acids Res 47: 11807-11825, 2019.

50. de Melo IS, Iglesias C, Benítez-Rondán A, Medina F, Martínez-Barberá JP and Bolívar J: NOA36/ZNF330 is a conserved cystein-rich protein with proapoptotic activity in human cells. Biochim Biophys Acta 1793: 1876-1885, 2009.
51. Willis S, Sun Y, Abramovitz M, Fei T, Young B, Lin X, Ni M, Achua J, Regan MM, Gray KP, et al: High expression of FGD3, a putative regulator of cell morphology and motility, is prognostic of favorable outcome in multiple cancers. JCO Precis Oncol 1: PO.17.00009, 2017.

52. Renda I, Bianchi S, Vezzosi V, Nori J, Vanzi E, Tavella K and Susini T: Expression of FGD3 gene as prognostic factor in young breast cancer patients. Sci Rep 9: 15204, 2019.

53. Vidigal JA and Ventura A: The biological functions of miRNAs: Lessons from in vivo studies. Trends Cell Biol 25: 137-147, 2015.

54. Peng $\mathrm{Y}$ and Croce CM: The role of microRNAs in human cancer. Signal Transduct Target Ther 1: 15004, 2016.

55. Bartel DP: Metazoan MicroRNAs. Cell 173: 20-51, 2018.

56. Michael IP, Saghafinia S and Hanahan D: A set of microRNAs coordinately controls tumorigenesis, invasion, and metastasis. Proc Natl Acad Sci USA 116: 24184-24195, 2019.

57. Cherone JM, Jorgji V and Burge CB: Cotargeting among microRNAs in the brain. Genome Res 29: 1791-1804, 2019.

58. Dong J, Xiao D, Zhao Z, Ren P, Li C, Hu Y, Shi J, Su H, Wang L, Liu H, et al: Epigenetic silencing of microRNA-137 enhances ASCT2 expression and tumor glutamine metabolism. Oncogenesis 6: e356, 2017.

59. Zhang Z, Liu R, Shuai Y, Huang Y, Jin R, Wang X and Luo J: ASCT2 (SLC1A5)-dependent glutamine uptake is involved in the progression of head and neck squamous cell carcinoma. Br J Cancer 122: 82-93, 2020.

60. Wu P, Xiao Y, Guo T, Wang Y, Liao S, Chen L and Liu Z: Identifying miRNA-mRNA pairs and novel miRNAs from hepatocelluar carcinoma mirnomes and TCGA database. J Cancer 10: 2552-2559, 2019

61. Nuzzo S, Catuogno S, Capuozzo M, Fiorelli A, Swiderski P, Boccella S, de Nigris F and Esposito CL: Axl-targeted delivery of the oncosuppressor miR-137 in non-small-cell lung cancer. Mol Ther Nucleic Acids 17: 256-263, 2019.

62. Drago-García D, Espinal-Enríquez J and Hernández-Lemus E: Network analysis of EMT and MET micro-RNA regulation in breast cancer. Sci Rep 7: 13534, 2017.

63. Schneider E, Pochert N, Ruess C, MacPhee L, Escano L, Miller C, Krowiorz K, Delsing Malmberg E, Heravi-Moussavi A, Lorzadeh A, et al: MicroRNA-708 is a novel regulator of the Hoxa9 program in myeloid cells. Leukemia 34: 1253-1265, 2020.

64. Looney AM, Nawaz K and Webster RM: Tumor-agnostic therapies. Nat Rev Drug Discov 19: 383-384, 2020.

65. Astras G, Papagiannopoulos CI, Kyritsis KA, Markitani C and Vizirianakis IS: Pharmacogenomic testing to guide personalized cancer medicine decisions in private oncology practice: A case study. Front. Oncol 10: 521, 2020.

66. Gu Z, Eils R and Schlesner M: Complex heatmaps reveal patterns and correlations in multidimensional genomic data. Bioinformatics 32: 2847-2849, 2016. Attribution-NonCommercial-NoDerivatives 4.0 International (CC BY-NC-ND 4.0) License. 